\title{
Equine Lentivirus Group
}

National Cancer Institute

\section{Source}

National Cancer Institute. Equine Lentivirus Group. NCI Thesaurus. Code C117713.

A non-taxonomic grouping of lentivuruses that infect horses, asses, and zebras. 